\title{
Histone chaperone FACT and curaxins: effects on genome structure and function
}

\author{
Han-Wen Chang ${ }^{1}$, Ekaterina V. Nizovtseva ${ }^{1}$, Sergey V. Razin ${ }^{2,3}$, Tim Formosa $^{4}$, Katerina V. Gurova ${ }^{5}$, Vasily \\ M. Studitsky1,3
}

${ }^{1}$ Cancer Epigenetics Program, Fox Chase Cancer Center, Philadelphia, PA 19111, USA.

${ }^{2}$ Institute of Gene Biology RAS, Moscow 119334, Russia.

${ }^{3}$ Biology Faculty, Lomonosov Moscow State University, Moscow 119992, Russia.

${ }^{4}$ Department of Biochemistry, University of Utah School of Medicine, Salt Lake City, Utah 84132, USA.

${ }^{5}$ Department of Cell Stress Biology, Roswell Park Cancer Institute, Buffalo, NY 14263, USA.

Correspondence to: Prof. Vasily M. Studitsky, Cancer Epigenetics Program, Fox Chase Cancer Center, 333 Cottman Ave., Philadelphia, PA 19111, USA. E-mail: vasily.studitsky@fccc.edu

How to cite this article: Chang HW, Nizovtseva EV, Razin SV, Formosa T, Gurova KV, Studitsky VM. Histone chaperone FACT and curaxins: effects on genome structure and function. $J$ Cancer Metastasis Treat 2019;5:78.

http://dx.doi.org/10.20517/2394-4722.2019.31

Received: 2 Sep 2019 First Decision: 4 Nov 2019 Revised: 12 Nov 2019 Accepted: 14 Nov 2019 Published: 29 Nov 2019

Science Editor: Godefridus J. Peters Copy Editor: Jing-Wen Zhang Production Editor: Tian Zhang

\begin{abstract}
The histone chaperone facilitates chromatin transcription (FACT) plays important roles in essentially every chromatin-associated process and is an important indirect target of the curaxin class of anti-cancer drugs. Curaxins are aromatic compounds that intercalate into DNA and can trap FACT in bulk chromatin, thus interfering with its distribution and its functions in cancer cells. Recent studies have provided mechanistic insight into how FACT and curaxins cooperate to promote unfolding of nucleosomes and chromatin fibers, resulting in genomewide disruption of contact chromatin domain boundaries, perturbation of higher-order chromatin organization, and global dysregulation of gene expression. Here, we discuss the implications of these insights for cancer biology.
\end{abstract}

Keywords: Genomics, transcription, nucleosomes, chromatin structure

\section{INTRODUCTION}

Facilitates chromatin transcription (FACT) is a highly conserved histone chaperone that participates in multiple physiological processes ${ }^{[1]}$ including transcription ${ }^{[2-8]}$, DNA replication ${ }^{[9-13]}$, DNA repair ${ }^{[14-17]}$,

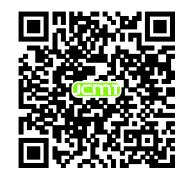


and centromere function ${ }^{[18,19]}$. FACT is a heterodimer of the suppressor of Ty 16 (Spt16) protein and the structure-specific recognition protein 1 (SSRP1) in mammals or the polymerase one binding protein 3 (Pob3) in yeast ${ }^{[1,8,9,20,21]}$. Unlike human SSRP1, the yeast Pob3 lacks the high mobility group B1 (HMGB1) DNA-binding domain; however, yeast FACT functionally interacts with the non-histone protein 6 (Nhp6) that consists of a single HMG box ${ }^{[22-24]}$. Both Spt16 and SSRP1 are able to interact with $\mathrm{H}_{2} \mathrm{~A} / \mathrm{H}_{2} \mathrm{~B}$ dimers and $\mathrm{H}_{3} / \mathrm{H} 4$ tetramers ${ }^{[20,25-29]}$. FACT is involved in both assembly and disassembly of nucleosomes, as well as in nucleosome unfolding that is dependent on its interactions with other factors. Single-particle Förster resonance energy transfer (spFRET) microscopy studies demonstrated that in the presence of Nhp6 protein yeast FACT can induce large-scale, reversible, and ATP-independent unfolding (reorganization) of individual nucleosomes that involves uncoiling of nucleosomal DNA ${ }^{[30,31]}$. In contrast, in the absence of Nhp6 protein, FACT can stabilize nucleosomes lacking the unstructured histone tails ${ }^{[32]}$. The Spt16 and SSRP1 subunits of FACT also contain additional intrinsic DNA-binding sites ${ }^{[33,34]}$. SSRP1 can bind the left-handed Z-form of DNA ${ }^{[35]}$. Notably, SSRP1 is usually phosphorylated by casein kinase 2, which inhibits its DNA binding activity ${ }^{[36,37]}$; the phosphorylated FACT can only bind destabilized nucleosomes or hexasomes ${ }^{[22,38]}$.

Curaxins are carbazole-based compounds that intercalate into DNA and alter the physical properties of both DNA and chromatin without causing structural damage (i.e., phosphodiester bond breaks or chemical modifications $)^{[34,39,40]}$. These drugs inhibit the activities of FACT, which has particularly severe consequences in cancer cells ${ }^{[35]}$. The curaxins CBL0137 and CBL0100 have been demonstrated to have broad anticancer activity in many different models ${ }^{[35,41-45]}$. Clinical candidate CBL0137 has higher metabolic stability and water solubility, and efficiently inhibits growth of various cancers in preclinical models ${ }^{[35]}$. CBL0100 is more biologically active but is rarely used in animal studies due to its lower solubility and higher toxicity. Curaxins were first identified in a screen for small molecule compounds capable of simultaneously inhibiting NF- $\mathrm{B}$, activating $\mathrm{p} 53$, and preferentially killing tumor cells ${ }^{[35]}$. Curaxins induce chromatin trapping (c-trapping) of FACT in less than 1 min after addition to cells, and strongly inhibit normal human FACT activities in vivo ${ }^{[35]}$. This c-trapping of FACT involves rapid formation of Z-DNA and binding of the SSRP1 subunit (z-trapping) ${ }^{[34]}$, as well as curaxin-dependent nucleosome unfolding resulting in binding of FACT to the disrupted nucleosomes (n-trapping) ${ }^{[31]}$. The anticancer activity of curaxins is highly dependent on c-trapping of $\mathrm{FACT}^{[31,34,35]}$. However, in addition to the c-trapping and activation of p53, curaxins also induce dramatic changes in chromatin structure (e.g., disruption of long-range chromatin interactions ${ }^{[46]}$, see below) and dysregulation of multiple cellular responses, including changes in transcription profiles ${ }^{[31,40]}$ and induction of transcription of repeats activates interferon (TRAIN) ${ }^{[47]}$.

This review focuses on recent studies of the roles of FACT and curaxins in gene expression and genome structure; other topics have been extensively covered in several excellent recent reviews ${ }^{[3,39,48]}$.

\section{ROLES OF FACT IN TRANSCRIPTION, REPLICATION, AND DNA DAMAGE REPAIR}

FACT is detected at transcribed regions of genes in multiple eukaryotes ${ }^{[40,49-51]}$. FACT is associated with both promoters and coding regions of transcribed genes ${ }^{[31,49,52,53]}$. Insertion of transposons (Ty elements) often interferes with the expression of nearby genes in Saccharomyces cerevisiae ${ }^{[54]}$. Depletion of yeast SPT16 causes cell cycle arrest in early G1 phase due to failure to transcribe cyclin genes, and less severe mutations cause the Spt- (suppressor of Ty) phenotype due to inappropriate activation of a cryptic promoter in Ty $1^{[54]}$. Yeast FACT and Nhp6 are also involved in maintaining nucleosome-depleted regions near many yeast promoters ${ }^{[52]}$. The nucleosome unfolding activity of yeast FACT working together with Nhp6 protein that has been detected in vitro likely plays a role in nucleosome destabilization and/or histone removal at promoter regions ${ }^{[2,30]}$. The in vitro studies of chromatin transcription by yeast RNA polymerase II (Pol II) have determined that FACT can facilitate Pol II transcript elongation through chromatin and nucleosome survival during this process by transiently interacting with the DNA binding surface of the $\mathrm{H} 2 \mathrm{~A} / \mathrm{H} 2 \mathrm{~B}$ 
dimers ${ }^{[9]}$. However, depletion of FACT only minimally affects transcript elongation rates in vivo, while numerous studies have shown that FACT is critically involved in maintaining the nucleosomal structure of the genome ${ }^{[6,955-59]}$. Thus, depletion of FACT in yeast causes accumulation of short transcripts from normal transcription start sites and cryptic transcripts initiated intragenically ${ }^{[56-59]}$. Yeast FACT also plays important roles in facilitating efficient replication and nucleosome assembly/disassembly during this process ${ }^{[14,15]}$. FACT occupancy is increased at DNA damage sites after different stresses, including UVinduced damage ${ }^{[17,60,61]}$, oxidative damage ${ }^{[19]}$, and single- and double-strand breaks ${ }^{[62,63]}$. FACT also plays roles in recovery from transcription arrest ${ }^{[6]]}$, replication fork stalling ${ }^{[64]}$, and R-loop formation ${ }^{[65]}$. The FACT subunit SSRP1 is able to detect non-B form DNA structures, and this activity might be important for inducing cell signaling cascades in response to these perturbations ${ }^{[34]}$.

FACT is essential for normal embryonic development. Ssrp1 knockout mice demonstrated early embryonic lethality at the blastocyst stage $(3.5 \mathrm{dpc})$, and ES cells derived from the Ssrp1-null blastocysts could not be propagated in vitro ${ }^{[66]}$. Defects in development were also observed in zebrafish with a mutant $S s r p 1^{[67]}$ and in plants with reduced levels of SSRP1 and SPT16 proteins ${ }^{[68,69]}$. Several studies also revealed that FACT subunits play specific roles in mammalian cell differentiation and considerably modify gene expression profiles during muscle cell differentiation ${ }^{[70]}$ and in vitro differentiation of human mesenchymal stem cells ${ }^{[71]}$. Although FACT can be involved in gene transcription, it is not ubiquitously expressed and therefore is not a required core component of the transcription machinery. The levels of FACT expression are higher in undifferentiated and proliferating cells, but are very low in most differentiated mammalian cells $^{[72-75]}$. Importantly, both curaxin treatment and (CRISPR)-mediated deletion of the FACT subunit Spt16 block induction of pluripotency without affecting the viability or proliferation of fibroblasts ${ }^{[76]}$. In tumor cells, both FACT subunits are highly expressed, and their higher levels correlate with poor prognosis in several tumor types ${ }^{[35,74,77-80]}$. Thus, FACT plays a more important role in cancer cells than in normal cells, making it a target for cancer therapy. The FACT complex is also involved in HIV integration into the host genome $^{[81]}$ and viral gene transcription ${ }^{[82,83]}$, suggesting that FACT could serve as a target for HIV therapy as well. The mechanistic studies of FACT activity described below therefore provide insights that could have clinical significance.

\section{FACT-NUCLEOSOME INTERACTIONS}

FACT does not bind stably to intact nucleosomes and does not affect nucleosome structure unless it is assisted by additional factors. The HMGB1-domain protein Nhp6 provides this nucleosome-destabilizing activity in vitro and in vivo for yeast FACT, and also supports nucleosome reorganization by human FACT in vitro ${ }^{[2]}$. The HMGB1 domain of SSRP1 does not appear to be adequate to promote uncoiling of the DNA from intact nucleosomes, suggesting that it may function primarily during nucleosome assembly ${ }^{[2,31,55]}$. Curaxins can distort DNA shape and this distortion promotes trapping of FACT in chromatin and changes chromatin compaction ${ }^{[31,46]}$. We propose that either HMGB proteins or curaxins weaken the histone-DNA contacts near the entry/exit sites of nucleosomal DNA and thus initiate a series of binding events by FACT domains that can lead to full reorganization of the nucleosome [Figure 1]. In this model, DNA distortion exposes the initial set of binding sites for the CTDs of each subunit of FACT, resulting in similar reorganization of the nucleosome independent of the initiating factor (curaxin or HMGB binding). In the reverse reaction leading to nucleosome assembly, Nhp6 or the intrinsic HMGB domain of SSRP1 can assist in stabilizing bent forms of the DNA to promote formation of the canonical nucleosome, resulting in release of FACT. Curaxins do not provide this assistance, instead leading to persistent binding of FACT and trapping it in chromatin (see below).

\section{CURAXIN-INDUCED TRAPPING OF FACT ON CHROMATIN}

Computational modeling and circular dichroism studies suggest that curaxins intercalate between DNA base pairs and the side chains of the molecule bind to both major and minor grooves of $\mathrm{DNA}^{[34,39]}$. Intercalation of curaxins in DNA causes an increase in the distance between the DNA base pairs, resulting 


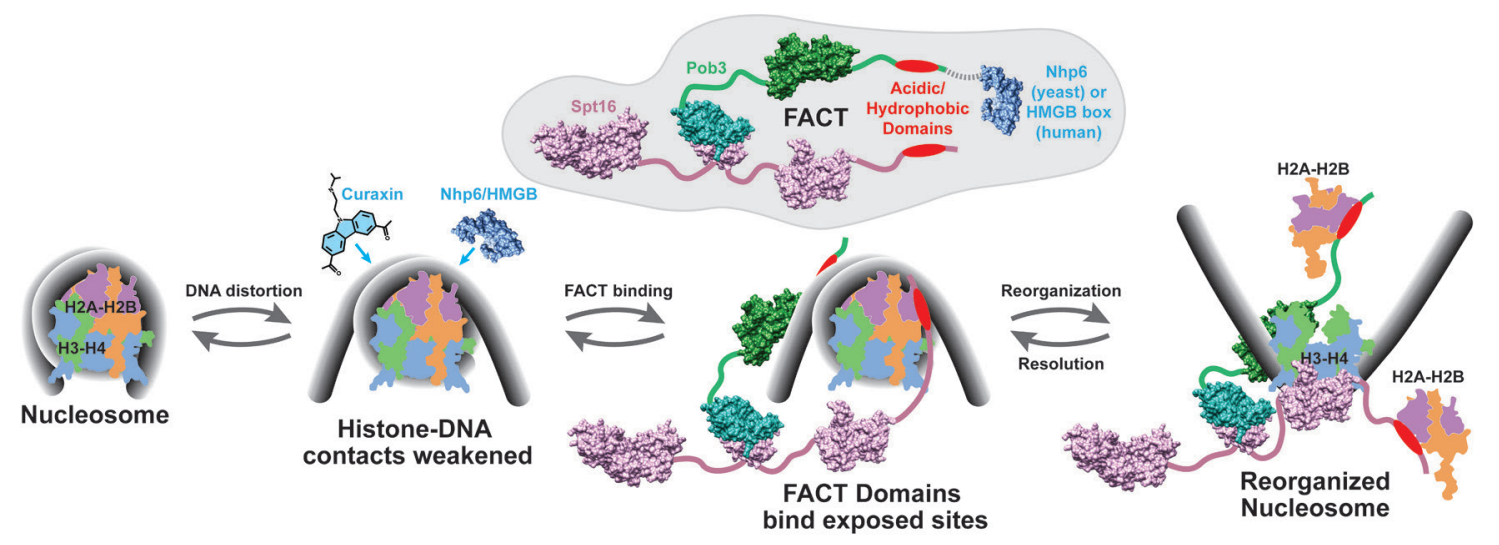

Figure 1. Nhp6 or curaxins can initiate uncoiling of nucleosomal DNA to promote reorganization by FACT. Either Nhp6/HMGB or curaxins can weaken the histone: DNA contacts near the entry/exit points of the nucleosomal DNA, exposing the binding site in $\mathrm{H} 2 \mathrm{~A}-\mathrm{H} 2 \mathrm{~B}$ dimers for the acidic/hydrophobic anchor sequences found in the C-terminal domains of both subunits of FACT ${ }^{[1]}$. The DNA distortion initiates a stepwise series of binding events by different domains of FACT that ultimately leads to uncoiling of the DNA to make the reorganized form of the nucleosome. Each step is reversible, providing a pathway for assembly of nucleosomes. Structures of the domains of FACT are shown, with the HMGB domain of SSRP1 represented by Nhp6 (modified from Figure $8^{[2]}$ ). FACT: facilitates chromatin transcription; Nhp6: non-histone protein 6; HMGB: high mobility group B; Spt16: suppressor of ty 16; Pob3: polymerase one binding protein 3

in unwinding of the DNA helix and a change in the DNA topology ${ }^{[34]}$. In the presence of curaxins, the nucleosome structure is destabilized in a dose- and time-dependent manner ${ }^{[31,34,39,40]}$. In cells, CBL0137 does not cause histone loss or nucleosome disassembly at concentrations lower than $3 \mu \mathrm{mol} / \mathrm{L}$ during a short time period (<20 min in cancer cells). However, histone loss is detectable in cells treated with $\geq 3 \mu \mathrm{mol} / \mathrm{L}$ CBL0137, and chromatin structure is destabilized when the concentration is $>5 \mu \mathrm{mol} / \mathrm{L}^{[34]}$. Similarly, CBL0137 and CBL0100 cause only minimal disruption of nucleosomes in cell free conditions at concentrations $\leq 2.5 \mu \mathrm{mol} / \mathrm{L}$ after incubation for several minutes ${ }^{[31,34]}$, while nucleosomes become unstable and are partially disassembled after incubation for a longer time period $(>1 \mathrm{~h})^{[31]}$.

Although at concentrations $\leq 2.5 \mu \mathrm{mol} / \mathrm{L}$ curaxins only minimally affect nucleosome structure, they significantly alter the structure of the internucleosomal linker DNA, increasing the average distance between the DNA regions entering and exiting a nucleosome ${ }^{[46]}$. On defined nucleosomal arrays the change in the structure of linker DNA is translated into a considerable decrease in the probability of long-range interactions, impacting enhancer-promoter communication (EPC) in vitro. As a result, enhancer-dependent transcription is strongly inhibited by curaxins; no direct effect on the activity of the transcriptional machinery itself was observed ${ }^{[46]}$. In agreement with the in vitro data, enhancer-dependent transcription is preferentially inhibited by curaxins in cells ${ }^{[46]}$.

Curaxin treatment has been shown to cause FACT (detected by fluorescence microscopy and chromatin immunoprecipitation) in cancer cells to disperse from transcribed regions and become trapped in other regions within $1 \mathrm{~min}^{[31,34]}$. This FACT redistribution results in genome-wide changes in FACT-dependent transcription profiles ${ }^{[31]}$. This phenomenon was recapitulated using a highly purified Pol II transcription system and positioned mononucleosomes in vitro ${ }^{[31]}$. Curaxins inhibit human (hFACT) action during chromatin transcription in vitro by trapping FACT complexes on competitor nucleosomes. Under conditions where hFACT and curaxins alone had minimal effects on nucleosomes in spFRET assay, they induced extensive, reversible uncoiling of nucleosomal DNA (nucleosome unfolding or reorganization) when combined, demonstrating highly synergistic action ${ }^{[31]}$. The non-transcribed regions of the genome are in excess over transcribed regions in a human cell, so FACT trapping by unfolded nucleosomes (n-trapping) causes depletion of hFACT from active regions of chromatin ${ }^{[31]}$. Thus, FACT $\mathrm{n}$-trapping is an important mechanism behind the short-term curaxin action in cancer cells. 
Although hFACT induces extensive, reversible nucleosome unfolding in the presence of either Nhp6 protein or curaxins [Figure 1], the properties of these FACT-unfolded nucleosomes are different ${ }^{[2,31]}$. The hFACT-yNhp6-nucleosome forms a reversible complex that is stable during electrophoresis, while the hFACT-nucleosome complex formed in the presence of curaxins is stable in solution, but falls apart during PAGE ${ }^{[2,31]}$. Since Nhp6 and curaxins are expected to bind to similar, end-proximal regions of the nucleosomal DNA and produce similar unfolding of nucleosomes together with $\mathrm{FACT}^{[2,31]}$, the differences in stability of the complexes may be explained by the different structures/stabilities of Nhp6-nucleosome and curaxin-nucleosome complexes, or possibly by a direct effect of Nhp6 protein on the properties of hFACT.

Many DNA binding molecules other than curaxins also cause trapping of FACT on chromatin in cellfree systems ${ }^{[40]}$. However, to be potent in chromatin destabilization, small molecules need to be able to get to the nuclei and compete with histones and other chromatin components for DNA binding. The curaxins that were selected and optimized in a cell-based assay for p53 activation ${ }^{[84,85]}$ easily enter into cell nuclei and are strong DNA binders. They were also selected in cells which were not responsive to DNA damage after p53 activation; thus, compounds capable of causing DNA damage were eliminated from the analysis. Curaxins can cause DNA damage in some systems; e.g., they cause frame shift mutations in some strains of E. coli in the Ames test. However, the much higher proofreading efficiency of mammalian DNA polymerases probably mitigates this effect at low concentrations of curaxins and blocks replication at high concentrations of the drug. Curaxins are also potent inhibitors of topoisomerases ${ }^{[34]}$, but, in contrast to many known drugs that inhibit resolution of topoisomerase cleavage complexes, do not prevent cleavage itself: curaxins inhibit initiation of topoisomerase action, preventing the induction of DNA breaks. Most importantly, the toxicity of curaxins for cells does not depend on DNA damage, since DNA damage is undetectable in many types of tumor cells as they are being killed by curaxin treatment ${ }^{[35]}$.

In addition to the c-trapping of FACT, curaxins globally affect the three-dimensional organization of the genome in living cells ${ }^{[46]}$. Studies employing nucleosome arrays show that curaxins induce changes in the structure of internucleosomal linker DNA and unfolding of the chromatin fiber ${ }^{[46]}$. Hi-C analysis demonstrated that addition of curaxins to cancer cells partially disrupted the boundaries of topologically associated domains (TADs), inhibited spatial interactions within the TADs (over distances less than $600 \mathrm{~Kb}$ ), and enhanced spatial interactions over longer distances ${ }^{[46]}$. These alterations of the three-dimensional genome organization strongly affected enhancer-promoter interactions: only 30\% of the intradomain chromatin interactions annotated in control cells were maintained in CBL0137-treated cells. As a result, numerous new alternative, long-range interdomain interactions were detected ${ }^{[46]}$. Furthermore, genomic studies demonstrated that curaxins caused partial depletion of CCCTC-binding factor (CTCF) from its binding sites $^{[46]}$. Since CTCF contributes to maintaining the spatial organization of the mammalian genome ${ }^{[86]}$, depletion of CTCF from domain boundaries by curaxins likely contributed to the drastic changes observed in genome topology. The changes in chromatin structure, in turn, are expected to dramatically change gene regulation and the pattern of gene expression. Indeed, after CBL0137 treatment, gene expression profiles were drastically changed. These changes included downregulation of essential genes necessary for cell survival, including both normal and translocated versions of MYC family genes, which could explain the observed drop in cancer cell viability ${ }^{[46]}$. Thus, curaxins can be classified as epigenetic drugs that globally affect gene regulation and cause cancer cell death by targeting the three-dimensional organization of the genome within living cell nuclei.

\section{THE MECHANISM OF CURAXIN ACTION}

Taken together, the current studies suggest the following scenario for curaxin action in cancer cells [Figure 2]. In untreated cancer cells, FACT is present mostly in the nucleoplasm; only a small fraction of the complexes are transiently associated with nucleosomes perturbed by transcribing RNA polymerase. 
Time after addition of curaxins:

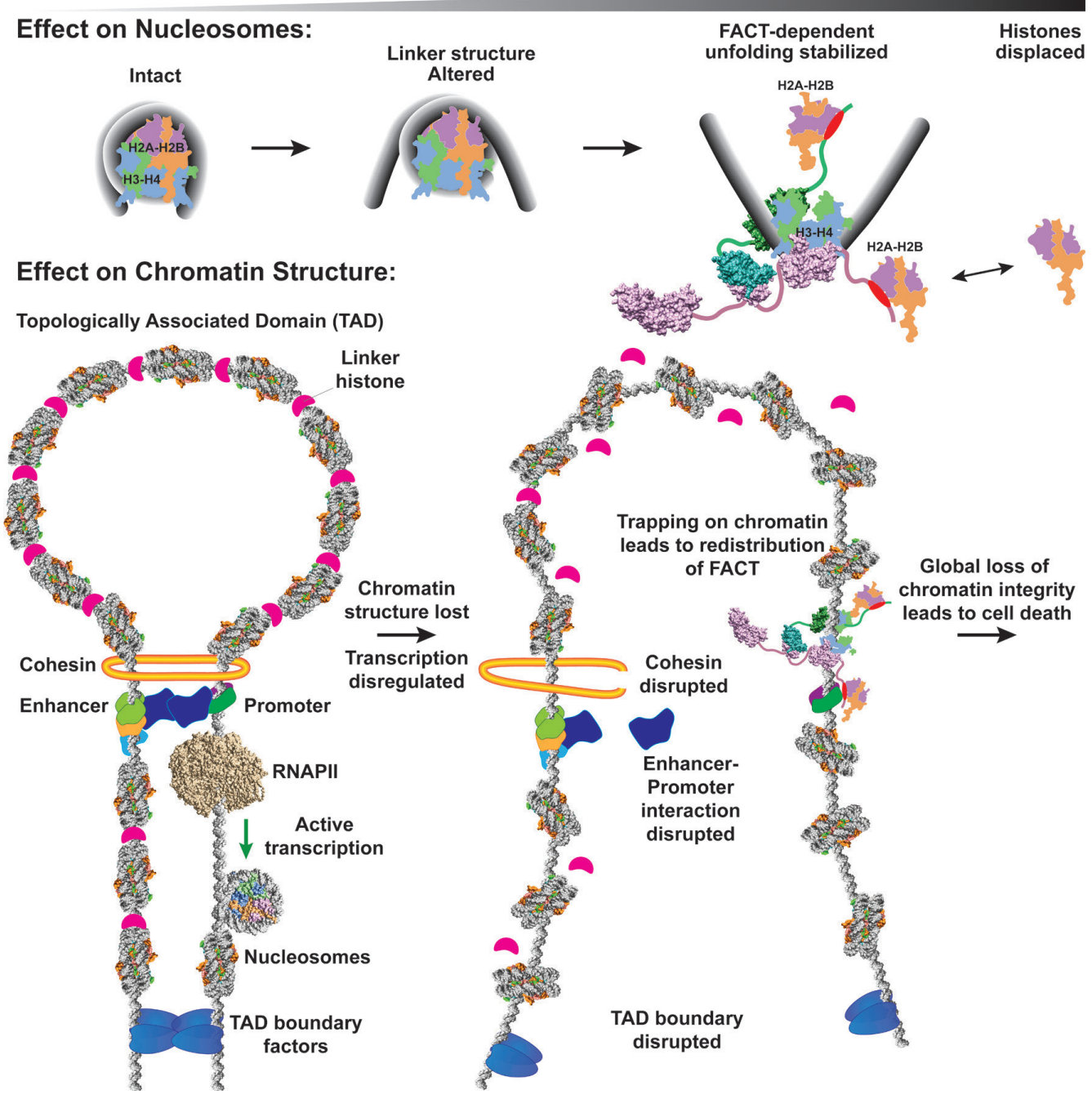

Figure 2. Curaxins affect different levels of chromatin structure. A proposed sequence of events during incubation of cancer cells with curaxins. Upper part: initially, curaxins induce time- and concentration-dependent FACT trapping on the unfolded nucleosomes. As curaxins intercalate into DNA, they induce progressive, FACT-dependent unfolding of nucleosomes that could eventually lead to eviction of core histones. Lower part: in parallel with the changes in the nucleosome structure, the higher-order chromatin structure is dramatically disturbed. Curaxins induce changes in the structure of internucleosomal linker DNA, releasing linker histones, unfolding the chromatin fiber, causing displacement of CTCF factor from DNA, and disrupting existing chromatin domain boundaries. Taken together, these changes in the structure of nucleosomes, chromatin fibers, and global three-dimensional chromatin organization lead to a large-scale loss of chromatin integrity and cancer cell death. Components are modeled as in Figure 1, with the addition of PDB 1Y1V (RNA polymerase II elongation complex structure). FACT: facilitates chromatin transcription; CTCF: CCCTC-binding factor

Curaxins rapidly induce changes in the structure of internucleosomal linker DNA and eviction of histone H1 from chromatin. The speed of this process is probably explained by the dynamic interaction of linker histones with chromatin ${ }^{[13]}$. FACT trapping in bulk chromatin is initiated at the same concentrations of curaxins (0.5-1 $\mu \mathrm{mol} / \mathrm{L}$ of CBL0137) that induce displacement of $\mathrm{H} 1$, and both processes occur at about the same time (a few seconds after addition of CBL0137). It is therefore possible that FACT participates in $\mathrm{H} 1$ eviction or prevents reassociation of $\mathrm{H} 1$ with linker DNA. Higher curaxin concentrations also displace core histones from chromatin.

Binding of FACT to bulk chromatin occurs at multiple genomic locations, but there is some preference for regions enriched for tandem dinucleotide AC/TG repeats known as mini- and microsatellites. These occur mostly at non-coding regions, which are dispersed throughout the human genome ${ }^{[87]}$. FACT binding and 
displacement of histone $\mathrm{H} 1$ contribute to unfolding of the chromatin fibers and also to largely reversible uncoiling of nucleosomal DNA (nucleosome unfolding). Since FACT is tightly bound to (trapped by) and unfolds nucleosomes genome-wide, FACT-dependent gene expression is globally affected, preferentially decreasing the viability of cancer cells that have a higher requirement for FACT. After a longer curaxin treatment, FACT-bound unfolded/destabilized nucleosomes are prone to disruption, possibly evicting core histones from the DNA. Nucleosome unfolding and histone eviction are likely to result in the release of negative DNA supercoiling that was constrained in intact nucleosomes and in formation of alternative DNA structures, such as Z-DNA, resulting in further FACT trapping.

In addition to affecting the structures of nucleosomes and chromatin fibers, curaxins globally alter genome topology in cells. Curaxins induce eviction of CTCF from DNA, thus disrupting domain boundaries and dramatically decreasing the number and type of chromatin loops that are characteristic of cancer cells. The latter effect is likely explained by the global unfolding of the chromatin fiber due to the altered geometry and topology caused by curaxin treatment. In turn, the changes in chromatin topology result in less efficient EPC, and in preferentially reduced expression levels of enhancer-dependent genes (e.g., Myc) and global alteration of the profiles of gene expression. The suppression of enhancer-dependent oncogenes strongly decreases the efficiency of cancer cell survival. This combined mechanism of curaxin action explains the high efficiency of the compounds and suggests novel therapeutic strategies targeting chromatin structure and spatial organization of the genome to alter gene expression profiles, thereby suppressing cancer cell growth.

\section{PERSPECTIVES}

Mutations in enzymes that "write", "read", or "erase" chromatin marks have emerged as a recurring theme in multiple types of cancer ${ }^{[88]}$. However, the mechanisms through which epigenetic changes benefit cancer cells remain poorly understood. Existing theories tend to focus on specific genes or pathways; that is, the effects of altering global properties of chromatin are usually attributed to altered expression of individual genes or groups of genes. However, discoveries such as "oncohistones", where mutations in core histones are associated with cancer ${ }^{[89]}$, raise the possibility that carcinogenesis results from a broad combination of defects occurring genome-wide.

The role of FACT in cancer, and the use of curaxins to combat cancers, face similar questions. Neither FACT nor curaxins have clear gene-specific effects, and yet the former preferentially supports the viability of aggressive cancer cells and the latter has clear anti-cancer activity. In one scenario, FACT and curaxins could function through a common mechanism by destabilizing nucleosomes. In this model, FACT might enhance global expression of genes, including a set that is important for maintaining excessive proliferation, and curaxins extend this activity past the point where it is advantageous. However, recent results suggest instead that FACT and curaxins might have opposing effects on nucleosomes, with curaxins promoting unwrapping of DNA from the core ${ }^{[34]}$, while FACT promotes survival of nucleosomes by tethering the components together and promoting reassembly ${ }^{[29]}$. As FACT is capable of both destabilizing and stabilizing nucleosomes, understanding how it promotes survival of cancer cells and how curaxins oppose this activity will ultimately require better understanding of how global nucleosome stability contributes to cancer cell progression and viability.

Pervasive cryptic transcription has been detected in multiple types of cancer ${ }^{[90-92]}$, suggesting that the DNA is more accessible to transcription machinery in tumor cells than it is in normal cells, as expected if the nucleosomal barrier is globally weakened. In this case, the role of FACT in stabilizing and restoring nucleosomes would be critical to allowing tumor cells to maintain chromatin. Curaxins would then interfere with this process, creating an excessive burden of disrupted chromatin, eventually leading to cell death. Then, the next questions to answer are: Why are nucleosomes less stable in tumors, thereby 
creating greater need for FACT activity? Do elevated transcription and replication decrease the stability of nucleosomes, or do tumor cells promote destabilization through an unknown mechanism to provide advantages such as easier reorganization of the three-dimensional structure of the genome or easier transitions among gene expression profiles? What is the relationship between nucleosome stability and three-dimensional chromatin structure? While it might seem obvious that loss of chromatin structure is disadvantageous, is this because of toxicity of the free histones that are produced by chromatin disassembly or is it the result of inappropriate exposure of DNA? Cancer cells survive by unleashing the beasts of excessive proliferation and metastasis that are normally constrained within chromatin; how do FACT and curaxins alter the balance between taking advantage of these dangerous forces to become cancerous and being overtaken by them?

\section{DECLARATIONS}

\section{Authors' contributions}

Wrote the initial draft of the manuscript and finalized it: Chang HW

Wrote parts of the manuscript: Nizovtseva EV, Razin SV

Wrote parts of the manuscript and prepared the final figures: Formosa $\mathrm{T}$

Wrote parts of the manuscript: Gurova KV

Designed the review and the figures, wrote parts of the manuscript and finalized it: Studitsky VM

\section{Availability of data and materials}

Not applicable.

\section{Financial support and sponsorship}

This work was supported by the National Cancer Institute (R01CA197967 to Gurova KV and R21CA220151 to Studitsky VM), the National Institute of General Medical Sciences (R01 GM119398 to Studitsky VM and R01 GM064649 to Formosa T), and by the Russian Science Foundation (19-74-30003).

\section{Conflicts of interest}

Gurova KV obtained a research grant and consulting payments from Incuron, Inc. The remaining authors declared that there are no conflicts of interest.

\section{Ethical approval and consent to participate}

Not applicable.

\section{Consent for publication}

Not applicable.

\section{Copyright}

(c) The Author(s) 2019.

\section{REFERENCES}

1. Gurova K, Chang HW, Valieva ME, Sandlesh P, Studitsky VM. Structure and function of the histone chaperone FACT - resolving FACTual issues. Biochim Biophys Acta Gene Regul Mech 2018; Epub ahead of print. doi: 10.1016/j.bbagrm.2018.07.008

2. LeRoy G, Orphanides G, Lane WS, Reinberg D. Requirement of RSF and FACT for transcription of chromatin templates in vitro. Science 1998;282:1900-4.

3. Orphanides G, LeRoy G, Chang CH, Luse DS, Reinberg D. FACT, a factor that facilitates transcript elongation through nucleosomes. Cell 1998;92:105-16.

4. Belotserkovskaya R, Oh S, Bondarenko VA, Orphanides G, Studitsky VM, et al. FACT facilitates transcription-dependent nucleosome alteration. Science 2003;301:1090-3.

5. Mason PB, Struhl K. The FACT complex travels with elongating RNA polymerase II and is important for the fidelity of transcriptional initiation in vivo. Mol Cell Biol 2003;23:8323-33. 
6. Saunders A, Werner J, Andrulis ED, Nakayama T, Hirose S, et al. Tracking FACT and the RNA polymerase II elongation complex through chromatin in vivo. Science 2003;301:1094-6.

7. Hsieh FK, Kulaeva OI, Patel SS, Dyer PN, Luger K, et al. Histone chaperone FACT action during transcription through chromatin by RNA polymerase II. Proc Natl Acad Sci U S A 2013;110:7654-9.

8. Orphanides G, Wu WH, Lane WS, Hampsey M, Reinberg D. The chromatin-specific transcription elongation factor FACT comprises human SPT16 and SSRP1 proteins. Nature 1999;400:284-8.

9. Wittmeyer J, Formosa T. The Saccharomyces cerevisiae DNA polymerase alpha catalytic subunit interacts with Cdc68/Spt16 and with Pob3, a protein similar to an HMG1-like protein. Mol Cell Biol 1997;17:4178-90.

10. Tan BC, Chien CT, Hirose S, Lee SC. Functional cooperation between FACT and MCM helicase facilitates initiation of chromatin DNA replication. EMBO J 2006;25:3975-85.

11. Abe T, Sugimura K, Hosono Y, Takami Y, Akita M, et al. The histone chaperone facilitates chromatin transcription (FACT) protein maintains normal replication fork rates. J Biol Chem 2011;286:30504-12.

12. Yang J, Zhang X, Feng J, Leng H, Li S, et al. The histone chaperone FACT contributes to DNA replication-coupled nucleosome assembly. Cell Rep 2016;14:1128-41.

13. Kurat CF, Yeeles JTP, Patel H, Early A, Diffley JFX. Chromatin controls DNA replication origin selection, lagging-strand synthesis, and replication fork rates. Mol Cell 2017;65:117-30.

14. Keller DM, Zeng X, Wang Y, Zhang QH, Kapoor M, et al. A DNA damage-induced p53 serine 392 kinase complex contains CK2, hSpt16, and SSRP1. Mol Cell 2001;7:283-92.

15. Krohn NM, Stemmer C, Fojan P, Grimm R, Grasser KD. Protein kinase CK2 phosphorylates the high mobility group domain protein SSRP1, inducing the recognition of UV-damaged DNA. J Biol Chem 2003;278:12710-5.

16. Heo K, Kim H, Choi SH, Choi J, Kim K, et al. FACT-mediated exchange of histone variant H2AX regulated by phosphorylation of H2AX and ADP-ribosylation of Spt16. Mol Cell 2008;30:86-97.

17. Charles Richard JL, Shukla MS, Menoni H, Ouararhni K, Lone IN, et al. FACT assists base excision repair by boosting the remodeling activity of RSC. PLoS Genet 2016;12:e1006221.

18. Murawska M, Ladurner AG. CENPs and sweet nucleosomes face the FACT. Trends Biochem Sci 2016;41:736-8.

19. Prendergast L, Muller S, Liu Y, Huang H, Dingli F, et al. The CENP-T/-W complex is a binding partner of the histone chaperone FACT. Genes Dev 2016;30:1313-26.

20. Winkler DD, Luger K. The histone chaperone FACT: structural insights and mechanisms for nucleosome reorganization. J Biol Chem 2011;286:18369-74.

21. Evans DR, Brewster NK, Xu Q, Rowley A, Altheim BA, et al. The yeast protein complex containing cdc68 and pob3 mediates corepromoter repression through the cdc68 N-terminal domain. Genetics 1998;150:1393-405.

22. Brewster NK, Johnston GC, Singer RA. A bipartite yeast SSRP1 analog comprised of Pob3 and Nhp6 proteins modulates transcription. Mol Cell Biol 2001;21:3491-502.

23. Formosa T, Eriksson P, Wittmeyer J, Ginn J, Yu Y, et al. Spt16-Pob3 and the HMG protein Nhp6 combine to form the nucleosome-binding factor SPN. EMBO J 2001;20:3506-17.

24. McCullough LL, Connell Z, Xin H, Studitsky VM, Feofanov AV, et al. Functional roles of the DNA-binding HMGB domain in the histone chaperone FACT in nucleosome reorganization. J Biol Chem 2018;293:6121-33.

25. Stuwe T, Hothorn M, Lejeune E, Rybin V, Bortfeld M, et al. The FACT Spt16 "peptidase" domain is a histone H3-H4 binding module. Proc Natl Acad Sci U S A 2008;105:8884-9.

26. Hondele M, Stuwe T, Hassler M, Halbach F, Bowman A, et al. Structural basis of histone H2A-H2B recognition by the essential chaperone FACT. Nature 2013;499:111-4

27. Kemble DJ, Whitby FG, Robinson H, McCullough LL, Formosa T, et al. Structure of the Spt16 middle domain reveals functional features of the histone chaperone FACT. J Biol Chem 2013;288:10188-94.

28. Kemble DJ, McCullough LL, Whitby FG, Formosa T, Hill CP. FACT disrupts nucleosome structure by binding H2A-H2B with conserved peptide motifs. Mol Cell 2015;60:294-306.

29. Wang T, Liu Y, Edwards G, Krzizike D, Scherman H, et al. The histone chaperone FACT modulates nucleosome structure by tethering its components. Life Sci Alliance 2018;1:e201800107.

30. Valieva ME, Armeev GA, Kudryashova KS, Gerasimova NS, Shaytan AK, et al. Large-scale ATP-independent nucleosome unfolding by a histone chaperone. Nat Struct Mol Biol 2016;23:1111-6.

31. Chang HW, Valieva ME, Safina A, Chereji RV, Wang J, et al. Mechanism of FACT removal from transcribed genes by anticancer drugs curaxins. Sci Adv 2018;4:eaav2131.

32. Valieva ME, Gerasimova NS, Kudryashova KS, Kozlova AL, Kirpichnikov MP, et al. Stabilization of nucleosomes by histone tails and by FACT revealed by spFRET microscopy. Cancers (Basel) 2017;9.

33. Hondele M, Ladurner AG. Catch me if you can: how the histone chaperone FACT capitalizes on nucleosome breathing. Nucleus 2013;4:443-9.

34. Safina A, Cheney P, Pal M, Brodsky L, Ivanov A, et al. FACT is a sensor of DNA torsional stress in eukaryotic cells. Nucleic Acids Res 2017;45:1925-45.

35. Gasparian AV, Burkhart CA, Purmal AA, Brodsky L, Pal M, et al. Curaxins: anticancer compounds that simultaneously suppress NFkappaB and activate p53 by targeting FACT. Sci Transl Med 2011;3:95ra74.

36. Li Y, Keller DM, Scott JD, Lu H. CK2 phosphorylates SSRP1 and inhibits its DNA-binding activity. J Biol Chem 2005;280:11869-75.

37. Tsunaka Y, Toga J, Yamaguchi H, Tate S, Hirose S, et al. Phosphorylated intrinsically disordered region of FACT masks its nucleosomal DNA binding elements. J Biol Chem 2009;284:24610-21.

38. Tsunaka Y, Fujiwara Y, Oyama T, Hirose S, Morikawa K. Integrated molecular mechanism directing nucleosome reorganization by human 
FACT. Genes Dev 2016;30:673-86.

39. Gurova KV. Chromatin stability as a target for cancer treatment. Bioessays 2019;41:e1800141.

40. Nesher E, Safina A, Aljahdali I, Portwood S, Wang ES, et al. Role of chromatin damage and chromatin trapping of FACT in mediating the anticancer cytotoxicity of DNA-binding small-molecule drugs. Cancer Res 2018;78:1431-43.

41. Burkhart C, Fleyshman D, Kohrn R, Commane M, Garrigan J, et al. Curaxin CBL0137 eradicates drug resistant cancer stem cells and potentiates efficacy of gemcitabine in preclinical models of pancreatic cancer. Oncotarget 2014;5:11038-53.

42. Carter DR, Murray J, Cheung BB, Gamble L, Koach J, et al. Therapeutic targeting of the MYC signal by inhibition of histone chaperone FACT in neuroblastoma. Sci Transl Med 2015;7:312ra176.

43. Dermawan JK, Hitomi M, Silver DJ, Wu Q, Sandlesh P, et al. Pharmacological targeting of the histone chaperone complex FACT preferentially eliminates glioblastoma stem cells and prolongs survival in preclinical models. Cancer Res 2016;76:2432-42.

44. Kim M, Neznanov N, Wilfong CD, Fleyshman DI, Purmal AA, et al. Preclinical validation of a single-treatment infusion modality that can eradicate extremity melanomas. Cancer Res 2016;76:6620-30.

45. Maluchenko NV, Chang HW, Kozinova MT, Valieva ME, Gerasimova NS, et al. Inhibiting the pro-tumor and transcription factor FACT: mechanisms. Mol Biol (Mosk) 2016;50:599-610.

46. Kantidze OL, Luzhin AV, Nizovtseva EV, Safina A, Valieva ME, et al. The anti-cancer drugs curaxins target spatial genome organization. Nat Commun 2019;10:1441.

47. Leonova K, Safina A, Nesher E, Sandlesh P, Pratt R, et al. TRAIN (transcription of repeats activates INterferon) in response to chromatin destabilization induced by small molecules in mammalian cells. Elife 2018;7.

48. Formosa T. The role of FACT in making and breaking nucleosomes. Biochim Biophys Acta 2013;1819:247-55.

49. Mylonas C, Tessarz P. Transcriptional repression by FACT is linked to regulation of chromatin accessibility at the promoter of ES cells. Life Sci Alliance 2018; 1:e201800085.

50. Kolundzic E, Ofenbauer A, Bulut SI, Uyar B, Baytek G, et al. FACT sets a barrier for cell fate reprogramming in caenorhabditis elegans and human cells. Dev Cell 2018;46:611-26.

51. Sandlesh P, Juang T, Safina A, Higgins MJ, Gurova KV. Uncovering the fine print of the CreERT2-LoxP system while generating a conditional knockout mouse model of Ssrp1 gene. PLoS One 2018;13:e0199785.

52. True JD, Muldoon JJ, Carver MN, Poorey K, Shetty SJ, et al. The modifier of transcription 1 (Mot1) ATPase and Spt16 histone chaperone co-regulate transcription through preinitiation complex assembly and nucleosome organization. J Biol Chem 2016;291:15307-19.

53. Tettey TT, Gao X, Shao W, Li H, Story BA, et al. A role for FACT in RNA polymerase II promoter-proximal pausing. Cell Rep 2019;27:3770-9.

54. Malone EA, Clark CD, Chiang A, Winston F. Mutations in SPT16/CDC68 suppress cis- and trans-acting mutations that affect promoter function in Saccharomyces cerevisiae. Mol Cell Biol 1991;11:5710-7.

55. Chen P, Dong L, Hu M, Wang YZ, Xiao X, et al. Functions of FACT in breaking the nucleosome and maintaining its integrity at the singlenucleosome level. Mol Cell 2018;71:284-93.

56. Kaplan CD, Laprade L, Winston F. Transcription elongation factors repress transcription initiation from cryptic sites. Science 2003;301:1096-9.

57. Duina AA, Rufiange A, Bracey J, Hall J, Nourani A, et al. Evidence that the localization of the elongation factor Spt16 across transcribed genes is dependent upon histone H3 integrity in Saccharomyces cerevisiae. Genetics 2007;177:101-12.

58. Jamai A, Puglisi A, Strubin M. Histone chaperone spt16 promotes redeposition of the original $\mathrm{h} 3-\mathrm{h} 4$ histones evicted by elongating RNA polymerase. Mol Cell 2009;35:377-83.

59. Hainer SJ, Charsar BA, Cohen SB, Martens JA. Identification of mutant versions of the Spt16 histone chaperone that are defective for transcription-coupled nucleosome occupancy in saccharomyces cerevisiae. G3 (Bethesda) 2012;2:555-67.

60. Keller DM, Lu H. p53 serine 392 phosphorylation increases after UV through induction of the assembly of the CK2.hSPT16.SSRP1 complex. J Biol Chem 2002;277:50206-13.

61. Dinant C, Ampatziadis-Michailidis G, Lans H, Tresini M, Lagarou A, et al. Enhanced chromatin dynamics by FACT promotes transcriptional restart after UV-induced DNA damage. Mol Cell 2013;51:469-79.

62. Kari V, Shchebet A, Neumann H, Johnsen SA. The H2B ubiquitin ligase RNF40 cooperates with SUPT16H to induce dynamic changes in chromatin structure during DNA double-strand break repair. Cell Cycle 2011;10:3495-504.

63. Gao Y, Li C, Wei L, Teng Y, Nakajima S, et al. SSRP1 cooperates with PARP and XRCC1 to facilitate single-strand DNA break repair by chromatin priming. Cancer Res 2017;77:2674-85.

64. O'Donnell AF, Brewster NK, Kurniawan J, Minard LV, Johnston GC, et al. Domain organization of the yeast histone chaperone FACT: the conserved N-terminal domain of FACT subunit Spt16 mediates recovery from replication stress. Nucleic Acids Res 2004;32:5894-906.

65. Herrera-Moyano E, Mergui X, Garcia-Rubio ML, Barroso S, Aguilera A. The yeast and human FACT chromatin-reorganizing complexes solve R-loop-mediated transcription-replication conflicts. Genes Dev 2014;28:735-48.

66. Cao S, Bendall H, Hicks GG, Nashabi A, Sakano H, et al. The high-mobility-group box protein SSRP1/T160 is essential for cell viability in day 3.5 mouse embryos. Mol Cell Biol 2003;23:5301-7.

67. Koltowska K, Apitz H, Stamataki D, Hirst EM, Verkade H, et al. Ssrpla controls organogenesis by promoting cell cycle progression and RNA synthesis. Development 2013;140:1912-8.

68. Duroux M, Houben A, Ruzicka K, Friml J, Grasser KD. The chromatin remodelling complex FACT associates with actively transcribed regions of the Arabidopsis genome. Plant J 2004;40:660-71.

69. Lolas IB, Himanen K, Gronlund JT, Lynggaard C, Houben A, et al. The transcript elongation factor FACT affects Arabidopsis vegetative and reproductive development and genetically interacts with HUB1/2. Plant J 2010;61:686-97.

70. Lolis AA, Londhe P, Beggs BC, Byrum SD, Tackett AJ, et al. Myogenin recruits the histone chaperone facilitates chromatin transcription (FACT) to promote nucleosome disassembly at muscle-specific genes. J Biol Chem 2013;288:7676-87. 
71. Hossan T, Nagarajan S, Baumgart SJ, Xie W, Magallanes RT, et al. Histone chaperone SSRP1 is essential for Wnt signaling pathway activity during osteoblast differentiation. Stem Cells 2016;34:1369-76.

72. Hertel L, De Andrea M, Bellomo G, Santoro P, Landolfo S, et al. The HMG protein T160 colocalizes with DNA replication foci and is down-regulated during cell differentiation. Exp Cell Res 1999;250:313-28.

73. Xiang YY, Wang DY, Tanaka M, Igarashi H, Naito Y, et al. Expression of structure-specific recognition protein mRNA in fetal kidney and Fe-nitrilotriacetate-induced renal carcinoma in the rat. Cancer Lett 1996;106:271-8.

74. Garcia H, Fleyshman D, Kolesnikova K, Safina A, Commane M, et al. Expression of FACT in mammalian tissues suggests its role in maintaining of undifferentiated state of cells. Oncotarget 2011;2:783-96.

75. Safina A, Garcia H, Commane M, Guryanova O, Degan S, et al. Complex mutual regulation of facilitates chromatin transcription (FACT) subunits on both mRNA and protein levels in human cells. Cell Cycle 2013;12:2423-34.

76. Shen Z, Formosa T, Tantin D. FACT inhibition blocks induction but not maintenance of pluripotency. Stem Cells Dev 2018;27:1693-701.

77. Garcia H, Miecznikowski JC, Safina A, Commane M, Ruusulehto A, et al. Facilitates chromatin transcription complex is an "accelerator" of tumor transformation and potential marker and target of aggressive cancers. Cell Rep 2013;4:159-73.

78. Fleyshman D, Prendergast L, Safina A, Paszkiewicz G, Commane M, et al. Level of FACT defines the transcriptional landscape and aggressive phenotype of breast cancer cells. Oncotarget 2017;8:20525-42.

79. Hudson ME, Pozdnyakova I, Haines K, Mor G, Snyder M. Identification of differentially expressed proteins in ovarian cancer using highdensity protein microarrays. Proc Natl Acad Sci U S A 2007;104:17494-9.

80. Koman IE, Commane M, Paszkiewicz G, Hoonjan B, Pal S, et al. Targeting FACT complex suppresses mammary tumorigenesis in Her2/ neu transgenic mice. Cancer Prev Res (Phila) 2012;5:1025-35.

81. Matysiak J, Lesbats P, Mauro E, Lapaillerie D, Dupuy JW, et al. Modulation of chromatin structure by the FACT histone chaperone complex regulates HIV-1 integration. Retrovirology 2017;14:39.

82. Gallastegui E, Millan-Zambrano G, Terme JM, Chavez S, Jordan A. Chromatin reassembly factors are involved in transcriptional interference promoting HIV latency. J Virol 2011;85:3187-202.

83. Jean MJ, Zhou D, Fiches G, Kong W, Huang H, et al. Curaxin CBL0137 has the potential to reverse HIV-1 latency. J Med Virol 2019;91:1571-6.

84. Gurova KV, Hill JE, Guo C, Prokvolit A, Burdelya LG, et al. Small molecules that reactivate p53 in renal cell carcinoma reveal a NFkappaB-dependent mechanism of p53 suppression in tumors. Proc Natl Acad Sci U S A 2005;102:17448-53.

85. Gurova KV, Hill JE, Razorenova OV, Chumakov PM, Gudkov AV. p53 pathway in renal cell carcinoma is repressed by a dominant mechanism. Cancer Res 2004;64:1951-8.

86. Dekker J, Mirny L. The 3D genome as moderator of chromosomal communication. Cell 2016;164:1110-21.

87. Katti MV, Ranjekar PK, Gupta VS. Differential distribution of simple sequence repeats in eukaryotic genome sequences. Mol Biol Evol 2001;18:1161-7.

88. Koschmann C, Nunez FJ, Mendez F, Brosnan-Cashman JA, Meeker AK, et al. Mutated chromatin regulatory factors as tumor drivers in cancer. Cancer Res 2017;77:227-33.

89. Nacev BA, Feng L, Bagert JD, Lemiesz AE, Gao J, et al. The expanding landscape of "oncohistone" mutations in human cancers. Nature 2019;567:473-8.

90. Barman P, Reddy D, Bhaumik SR. Mechanisms of antisense transcription initiation with implications in gene expression, genomic integrity and disease pathogenesis. Noncoding RNA 2019;5.

91. Modur V, Singh N, Mohanty V, Chung E, Muhammad B, et al. Defective transcription elongation in a subset of cancers confers immunotherapy resistance. Nat Commun 2018;9:4410.

92. Wade JT, Grainger DC. Spurious transcription and its impact on cell function. Transcription 2018;9:182-9. 